\title{
Incorporating sprouted chickpea flour in pasta increases brachial artery flow-mediated dilation
}

\author{
JA Bruno ${ }^{1}$, CH Feldman ${ }^{1}$ DW Konas ${ }^{2}$, AL Kerrihard ${ }^{1}$, EL Matthews ${ }^{3}$ \\ ${ }^{1}$ Department of Nutrition and Food Studies, Montclair State University, Montclair, NJ, USA \\ ${ }^{2}$ Department of Chemistry and Biochemistry, Montclair State University, Montclair, NJ, USA \\ ${ }^{3}$ Department of Exercise Science and Physical Education, Montclair State University, Montclair, NJ, USA
}

Received: March 11, 2019

Accepted: August 29, 2019

\begin{abstract}
Purpose: Consumption of alternative flours, such as sprouted chickpea flour, has shown increased popularity in recent years. Foods rich in antioxidants have been shown to influence brachial artery flow-mediated dilation (FMD), a non-invasive test of a crucial layer of the artery called the endothelium. Partially replacing the semolina flour in pasta with sprouted chickpea flour (SCF) may acutely affect endothelial function post-digestion. We sought to determine if FMD was higher, lower, or the same post-digestion of pasta made with $60 \%$ semolina flour and $40 \%$ SCF (SCF40) vs. post-digestion of pasta made with $100 \%$ semolina flour (SEM100, i.e., control). Methods: Trolox equivalent antioxidant capacity (TEAC) analysis was performed on the same flour samples. Healthy participants underwent a screening visit and two randomized controlled meal data collection visits (SCF40 and SEM100). At each data collection visit, participants consumed $255 \mathrm{~g}$ of pasta with butter. FMD was assessed $2-3 \mathrm{~h}$ after pasta consumption. Results: TEAC results showed that SCF40 $(2.031 \pm 0.096 \mathrm{mmol}$ trolox $/ 100 \mathrm{~g}$ sample $)$ had significantly greater antioxidant capacity than SEM100 $(1.736 \pm 0.046 \mathrm{mmol}$ trolox $/ 100 \mathrm{~g}$ sample; $p=0.02)$. Twenty-two healthy participants ( 5 men and 17 women; $26 \pm 2$ years, $66.6 \pm 2.3 \mathrm{~kg}, \mathrm{BMI}=24 \pm 1 \mathrm{~kg} / \mathrm{m}^{2}, \mathrm{SBP}=114 \pm 3 \mathrm{mmHg}$, $\mathrm{DBP}=75 \pm 2 \mathrm{mmHg}, \mathrm{HR}=74 \pm 3 \mathrm{BPM})$ were studied. FMD in the SCF40 condition $(10.3 \% \pm 1.2 \%)$ was greater than the SEM100 condition $(7.9 \% \pm 0.8 \%, p=0.02)$. Conclusion: These data suggest that partial substitution with sprouted chickpea flour in place of semolina flour in pasta acutely improves post-digestion FMD, which may be beneficial for cardiovascular health (ClinicalTrials.gov Identifier: NCT03801486).
\end{abstract}

Keywords: germinated, garbanzo, endothelial, vascular, antioxidant, dietary

\section{Introduction}

Endothelial dysfunction is a precursor to the development of atherosclerosis (8). Endothelial dysfunction can be caused by oxidative stress, which limits the bioavailability of nitric oxide (NO), which is a vasodilator (15). Oxidative stress can uncouple endothelial NO synthase (eNOS) from its cofactor or substrate leading to the production of superoxide instead of NO (7). Eating foods rich in dietary antioxidants is largely believed to reduce oxidative stress, and to be beneficial for endothelial function (15). Furthermore, a single meal high in antioxidants increases serum levels of antioxidants (5), which may cause positive acute effects on endothelial function (14). However, antioxidant supplementation had mixed results when examining effects on cardiovascular function (16), and can even impair vascular function in normal healthy adults (13), presumably due to the excessively high quantity of antioxidants in supplements.

\footnotetext{
Corresponding author: Evan L. Matthews, $\mathrm{PhD}$

Department of Exercise Science and Physical Education, Montclair State University

1 Normal Avenue, Montclair, NJ 07043, USA

Phone: +1 973655 3948; Fax: +1 973655 5461; E-mail: matthewse@montclair.edu
} 
Due to the presumed health benefits, the general public is seeking out alternative flour options (3), such as chickpea flour. However, it is unclear what the acute effects of this antioxidant-rich dietary substitution (2) may be on endothelial function in healthy adults. We hypothesized that a $40 \%$ substitution of semolina flour with sprouted chickpea flour (SCF40), or non-sprouted chickpea flour (non-SCF40), would increase the antioxidant capacity in vitro as measured by trolox equivalent antioxidant capacity (TEAC). Following the in vitro testing, we hypothesized that brachial artery flow-mediated dilation (FMD), a test of in vivo endothelial function, would be greater following the consumption of pasta prepared with SCF40 than $100 \%$ semolina flour (SEM100).

\section{Materials and Methods}

\section{TEAC assay}

Antioxidant potential was assessed using TEAC assay for SEM100, non-SCF40, and SCF40. As described in previous studies (1), TEAC was evaluated with minor modifications. Briefly, the hydrophilic fractions of $1.25 \mathrm{~g}$ of flour were obtained by extraction with $4: 1(\mathrm{v} / \mathrm{v})$ acetonewater solvents followed by removal of the solvents under reduced pressure. The resulting material was dissolved in 99.7:0.3 (v/v) water/formic acid to a final volume of $7.5 \mathrm{ml}$. A 0.10-mM 2,2-diphenyl-1-picrylhydrazyl (DPPH) solution was prepared in 4:1 (v/v) methanol $/ \mathrm{H}_{2} \mathrm{O}$. For the assay, $10 \mu \mathrm{l}$ of sample were added to $290 \mu \mathrm{l}$ of DPPH solution in wells of a VersaMax ELISA Microplate Reader (Sunnyvale, CA, USA). After $30 \mathrm{~min}$, the absorbance at $517 \mathrm{~nm}$ in each well was recorded. These values were correlated with a standard curve constructed using solutions of $0-5 \mathrm{mM}$ trolox in 1:1 (v/v) acetone-water and reported as mmol trolox equivalent/100 g sample. All TEAC values were determined in quadruplicate.

\section{Semolina and sprouted chickpea pasta protocols}

The chickpea sprouts were prepared in the Food Science Laboratory at Montclair State University using a protocol described previously (6). Four parts of sprouted chickpea flour were mixed with six parts of Caputo Semola Di Grano Duro Rimacinata (Naples, Italy) semolina flour to obtain SCF40. SCF40 was chosen as the experimental condition because no notable decrements in pasta quality were noted on preliminary assessment with up to $40 \%$ substitution of semolina with SCF, and because sprouted chickpea flour was found to have the highest antioxidant capacity among those assessed (see "Results"). An amount of $400 \mathrm{~g}$ of the SCF40 mixture was then mixed with $160 \mathrm{ml}$ of water to form a dough. The dough was then placed in a plastic wrap and left to sit for $10 \mathrm{~min}$ at room temperature. This dough was then formed into balls and placed into a KitchenAid Gourmet Pasta Press attachment of a KitchenAid machine using the rigatoni attachment (Benton Harbor, MI USA). The pasta was left in the refrigerator at $0.6^{\circ} \mathrm{C}$ for $24 \mathrm{~h}$. Before each participant visit, the pasta was placed in three cups of boiling water for $5 \mathrm{~min}$. The pasta was then weighed to $255 \mathrm{~g}$ in a plastic container and was combined with $21 \mathrm{~g}$ of salted butter for flavoring.

SEM100 pasta was prepared by combining $400 \mathrm{~g}$ of Caputo Semola Di Grano Duro Rimacinata semolina flour with $118 \mathrm{ml}$ of water to form a dough. The dough was then placed in a plastic wrap and left to sit for $10 \mathrm{~min}$ at room temperature. The remaining procedure was the same as the SCF40 pasta. 


\section{Study population}

The experimental protocols and the process for obtaining informed consent conformed to the provisions in the Declaration of Helsinki and were approved by the Institutional Review Board of Montclair State University. Informed consent was obtained from all individual participants included in the study. To enroll, participants needed to be 18-60 years of age, have no known cardiovascular disease, not be pregnant, and not have food allergies that would prevent them from being able to eat the study-related food. Thirty-four participants were enrolled, three dropped out following the screening, and two participants participated in the first data collection visit but did not complete the second. Therefore, there were 29 healthy adults participated in the entire study: 6 men and 23 women. Seven participants had visible arterial shifts during at least one recording making their data unusable. Therefore, only 22 participants (5 men and 17 women) are included in this analysis.

\section{Experimental protocol}

Participants reported to the Exercise Science Laboratory at Montclair State University for an initial screening visit. Baseline blood pressure, height, weight, and body composition were determined. Participants completed a medical questionnaire and consent form. Participants were excluded if they had diagnosed cardiovascular disease, hypertension, malignancy, diabetes mellitus, or renal impairment. Participants who used tobacco products and those taking any medications with known cardiovascular effects were asked to refrain from use $8 \mathrm{~h}$ prior to experimental and control visits.

\section{Experimental and control visits}

Prior to the consumption of pasta, the participants were asked to be in fasting for $4 \mathrm{~h}$; avoid caffeine for $12 \mathrm{~h}$; and avoid alcohol consumption, over-the-counter drug use, and strenuous physical activity for $24 \mathrm{~h}$. Participants consumed each pasta sample $2-3 \mathrm{~h}$ before FMD testing. This length of time is enough to allow the pasta to be digested and for the glucose to enter and be removed from the blood stream (4). No more than 7 days was allowed between the experimental and control visits, and the visits were scheduled for the same time of day and were done so in a balanced randomized order. The brachial artery FMD protocol was performed as done previously (9) using a 5-13 $\mathrm{MHz}$ linear phased array ultrasound transducer (GE Vivid i; Healthcare, Jiangsu, China).

\section{Statistical analysis}

TEAC assay trials (each $n=4$ ) were compared between three flour compositions (SEM100, non-SCF40, and SCF40) using a one-way analysis of variance (ANOVA). Dunnett's test with SEM100 as the control was used as the one-way ANOVA post-hoc test. Statistical power calculations determined that 20 participants would be needed to detect a $10 \%$ difference in FMD between conditions with $90 \%$ power and an $\alpha$ level of 0.05 . All measurements compared between the experimental and control visits were analyzed using a paired $t$-test. Data are expressed as mean $\pm \mathrm{SE}$.

\section{Results}

There was a significant effect for flour types in the TEAC analysis: SEM100, $1.74 \pm 0.05$; non-SCF40, $1.95 \pm 0.03$; and SCF40, $2.03 \pm 0.10 \mathrm{mmol}$ trolox/100 g sample (ANOVA, 
Table I. Cardiovascular measurements during control and experimental visits

\begin{tabular}{|l|c|c|c|}
\hline & SEM100 & SCF40 & $p$ value \\
\hline SBP $(\mathrm{mmHg})$ & $112 \pm 2$ & $111 \pm 2$ & 0.88 \\
\hline DBP $(\mathrm{mmHg})$ & $68 \pm 2$ & $68 \pm 1$ & 0.85 \\
\hline MAP $(\mathrm{mmHg})$ & $83 \pm 2$ & $83 \pm 2$ & 0.85 \\
\hline Heart rate $(\mathrm{bpm})$ & $68 \pm 2$ & $67 \pm 2$ & 0.62 \\
\hline Baseline artery diameter $(\mathrm{mm})$ & $3.05 \pm 0.15$ & $3.03 \pm 0.13$ & 0.79 \\
\hline FMD shear rate $(\mathrm{AUC})$ & $53,463 \pm 5,425$ & $48,591 \pm 6,423$ & 0.38 \\
\hline FMD dilation $(\Delta \mathrm{mm})$ & $0.23 \pm 0.02$ & $0.30 \pm 0.03$ & 0.02 \\
\hline FMD dilation $(\% \Delta)$ & $7.9 \pm 0.8$ & $10.3 \pm 1.2$ & 0.02 \\
\hline
\end{tabular}

Cardiovascular assessments are taken between the 100\% semolina pasta and $60 \%$ semolina pasta/40\% sprouted chickpea pasta visits. Values represent mean \pm SE. SEM100: 100\% semolina flour; SCF40: 40\% sprouted chickpea flour; SBP: systolic blood pressure; DBP: diastolic blood pressure; MAP: mean arterial pressure; FMD: flowmediated dilation; AUC: area under the curve
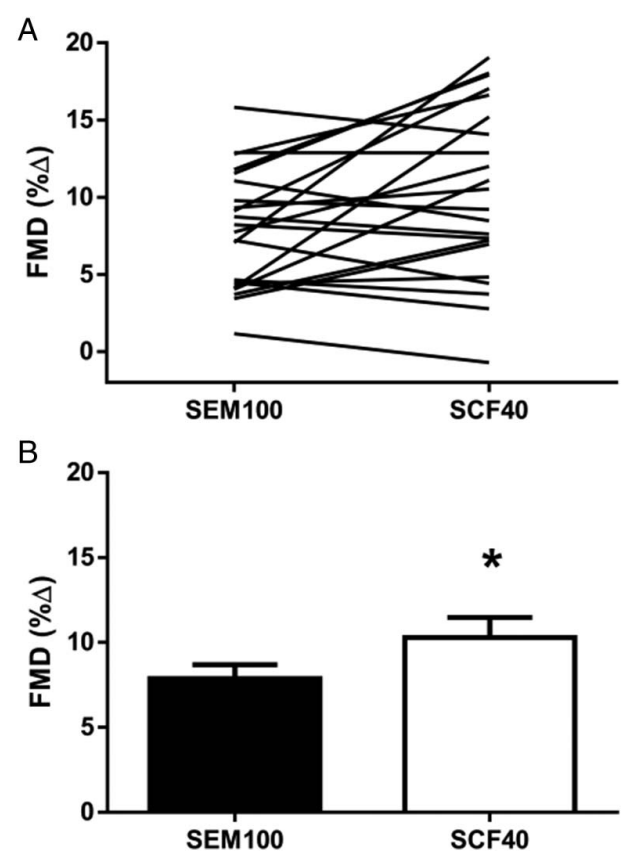

Fig. 1. Flow-mediated dilation (FMD). FMD assessment was greater in the $60 \%$ semolina flour and $40 \%$ sprouted chickpea flour (SCF40) condition than the $100 \%$ semolina flour (SEM100) control condition $(p=0.02)$. Panel A shows individual data and Panel B displays means $\pm \mathrm{SE}$

$p=0.03)$. Post-hoc testing found that SCF40 $(p=0.02)$ but not non-SCF40 $(p=0.08)$ had greater antioxidant potential than SEM100.

Data from the screening visit found that participants were generally young (age $=26 \pm$ 2 years), with normal body composition (height $=165.6 \pm 1.8 \mathrm{~cm}$, weight $=66.6 \pm 2.3 \mathrm{~kg}$, body fat $=25 \% \pm 2 \%, \mathrm{BMI}=24 \pm 1 \mathrm{~kg} / \mathrm{m}^{2}$ ) and normotensive [systolic blood pressure 
$(\mathrm{SBP})=114 \pm 3 \mathrm{mmHg}$, diastolic blood pressure $(\mathrm{DBP})=75 \pm 2 \mathrm{mmHg}$, mean arterial pressure $(\mathrm{MAP})=88 \pm 2 \mathrm{mmHg}$, heart rate $(\mathrm{HR})=74 \pm 3 \mathrm{bpm}]$

There were no differences between the blood pressure or HR values between visits (Table I). During the FMD protocol, baseline artery diameter and shear rate were not different between conditions (Table I). Both FMD assessed as a delta value and percent change from baseline (Fig. 1) were significantly greater in the SCF40 condition (Table I).

\section{Discussion}

The main findings of this study are that partial replacement (40\%) of semolina flour with sprouted chickpea flour increased antioxidant potential of the flour mixture and increased post-consumption FMD. This effect was observed in healthy young participants with no known cardiovascular disease. This is important as foods containing alternative flours are currently trending with the general public (3), and it is important to understand their effects. The current investigation demonstrates how a simple dietary substitution (i.e., partially removing semolina flour for sprouted chickpea flour) can have positive acute effects on cardiovascular function.

Others have found that increased levels of flavonoids increase FMD in a dose-dependent manner (10), presumably due to the antioxidant potential of flavonoids (12). Antioxidants help to quench free radicals, which may cause eNOS uncoupling due to oxidative stress (15). Diets rich in antioxidants are largely considered to reduce oxidative stress, and be beneficial for endothelial function (15). Conversely, antioxidant supplementation had mixed results when examining cardiovascular function (16), likely due to the excessively high quantity of antioxidants found in supplements.

Given the lack of blood antioxidant data in the current investigation, we must acknowledge the possibility of non-antioxidant mechanisms. The addition of chickpea flour decreases the glycemic index of pasta, which reduces the risk of hyperglycemia during peak absorption (4). However, the current investigation allowed 2-3 h for digestion to decrease the effect of blood glucose on FMD (4). Regardless, previous research suggests that a highcarbohydrate meal does not affect FMD (11).

\section{Conclusions}

The results of this study suggest that substituting $40 \%$ of the semolina flour in pasta with sprouted chickpea flour improves in vitro antioxidant capacity and post-consumption endothelial function in healthy adults. Thus, our results suggest that partially replacing traditional semolina flour with sprouted chickpea flour may be a beneficial and simple dietary intervention for improving cardiovascular health. Future longitudinal studies should be conducted to establish chronic benefits versus the acute benefits shown in this investigation.

\section{REFERENCES}

1. Brand-Williams W, Cuvelier M-E, Berset C: Use of a free radical method to evaluate antioxidant activity. LWTFood Sci. Technol. 28, 25-30 (1995)

2. Bruno J, Konas D, Matthews E, Feldman C, Pinsley K, Kerrihard A: Sprouted and non-sprouted chickpea flours: effects on sensory traits in pasta and antioxidant capacity. Polish J. Food Nutr. Sci. 69, 203-209 (2019) 
3. Future Market Insights (2018). Chickpea Flour Market: APEJ to Remain Dominant: Global Industry Analysis 2012-2016 \& Opportunity Assessment 2017-2026 [Online]. Available at: https://www.futuremarketinsights. com/reports/chickpea-flour-market

4. Goñi I, Valentín-Gamazo C: Chickpea flour ingredient slows glycemic response to pasta in healthy volunteers. Food Chem. 81, 511-515 (2003)

5. Jensen GS, Wu XL, Patterson KM, Barnes J, Carter SG, Scherwitz L, Beaman R, Endres JR, Schauss AG: In vitro and in vivo antioxidant and anti-inflammatory capacities of an antioxidant-rich fruit and berry juice blend. Results of a pilot and randomized, double-blinded, placebo-controlled, crossover study. J. Agric. Food Chem. 56, 8326-8333 (2008)

6. Khattak AB, Zeb A, Bibi N, Khalil SA, Khattak MS: Influence of germination techniques on phytic acid and polyphenols content of chickpea (Cicer arietinum L.) sprouts. Food Chem. 104, 1074-1079 (2007)

7. Luo S, Lei H, Qin H, Xia Y: Molecular mechanisms of endothelial NO synthase uncoupling. Curr. Pharm. Des. 20, 3548-3553 (2014)

8. Lüscher TF, Tanner FC, Tschudi MR, Noll G: Endothelial dysfunction in coronary artery disease. Annu. Rev. Med. 44, 395-418 (1993)

9. Matthews EL, Brian MS, Ramick MG, Lennon-Edwards S, Edwards DDG, Farquhar WB: High dietary sodium reduces brachial artery flow-mediated dilation in humans with salt-sensitive and salt-resistant blood pressure. J. Appl. Physiol. 118, 1510-5 (2015)

10. Monahan KD, Feehan RP, Kunselman AR, Preston AG, Miller DL, Lott MEJ: Dose-dependent increases in flow-mediated dilation following acute cocoa ingestion in healthy older adults. J. Appl. Physiol. 111, 1568-74 (2011)

11. Padilla J, Harris RA, Fly AD, Rink LD, Wallace JP: The effect of acute exercise on endothelial function following a high-fat meal. Eur. J. Appl. Physiol. 98, 256-262 (2006)

12. Pietta PG: Flavonoids as antioxidants. J. Nat. Prod. 63, 1035-1042 (2000)

13. Richardson RS, Donato AJ, Uberoi A, Wray DW, Lawrenson L, Nishiyama S, Bailey DM: Exercise-induced brachial artery vasodilation: role of free radicals. Am. J. Physiol. Heart Circ. Physiol. 292, H1516-522 (2007)

14. Sincer İ, Kurtoğlu E, Çoşkun FY, Aktürk S, Vuruşkan E, Düzen IV, Saraçoğlu E, Aktürk E, Hidayet Ş: Association between serum total antioxidant status and flow-mediated dilation in patients with systemic lupus erythematosus: an observational study. Anatol. J. Cardiol. 15, 913-918 (2015)

15. Varadharaj S, Kelly OJ, Khayat RN, Kumar PS, Ahmed N, Zweier JL: Role of dietary antioxidants in the preservation of vascular function and the modulation of health and disease. Front. Cardiovasc. Med. 4, 64 (2017)

16. Willcox BJ, Curb JD, Rodriguez BL: Antioxidants in cardiovascular health and disease: key lessons from epidemiologic studies. Am. J. Cardiol. 101, S75-S86 (2008) 